R. J. Cohen and W. T. Sullivan, III, eds.

\title{
Protection of Millimetre-Wave Astronomy
}

\author{
Masatoshi Ohishi \\ The National Astronomical Observatory, 2-21-1, Osawa, Mitaka,Tokyo, \\ 181-8588, Japan
}

\begin{abstract}
Development of radio technologies will lead to a serious conflict between millimetre-wave astronomy and telecommunication services. I describe characteristics of millimetre-wave astronomy and technical aspects related to radio astronomical observations. Three examples of possible interference to millimetre-wave astronomy are described. It is very important to advertise what millimetre-wave astronomy contributes to human culture and to get support from the non-astronomical community to keep the radio windows open and clean.
\end{abstract}

\section{Introduction}

Over the past quarter-century, astronomical studies in the millimetre and submillimetre wavebands have grown into one of the major disciplines of astronomy. These wavebands contain thousands of observable rotational molecular spectral lines, many of which are invaluable physical and chemical probes of the interstellar and circumstellar media. Furthermore, these bands contain the longwavelength emission of cool dust and the short-wavelength tail of synchrotron emission, both of which are important for establishing the spectrum and physical properties of the emitting source. Low energy phenomena produce emission in the millimetre wave spectral region. Interstellar clouds have kinetic temperatures of a few tens of Kelvins, and can excite the low-energy rotational transitions of gas molecules. The spectroscopic emission from these transitions can, in principle, yield the abundance, temperature and velocity structure of the emitting gas. To achieve unique solutions for these quantities, observations of multiple transitions of the same or different species are often required. For the purposes of this paper, I will define the $\mathrm{mm} / \mathrm{submm}$ bands as the frequency range from 30 to $1000 \mathrm{GHz}$ (wavelengths $10 \mathrm{~mm}$ to $300 \mu \mathrm{m}$ respectively).

\subsection{Science in the Millimetre-Wave Bands}

What happened just after the Big Bang and what is the destiny of our Universe? How do stars and galaxies form? How did our solar system form? Do planets like the earth exist around other stars? What role did the cosmos play in the development and evolution of life on earth? How do stars die and how is their material recycled into new stars? These fascinating questions - fundamental to our understanding of the Universe - are but a few that astronomers seek to answer. 
Radio astronomy utilizes the very latest radio technology to address such questions and to contribute to the cumulative knowledge of society. Recent research in radio astronomy has provided important information on the birth and death of stars, the detection of planets and proto-planetary systems around other stars, the formation of external galaxies just after the Big Bang, discoveries of black holes, discoveries of molecules and unexpectedly complex chemistry in space, to give but a few examples.

\subsection{Millimetre-Wave Radio Astronomy}

Millimetre-wave radio astronomy is a special branch of astronomy developed over the past quarter-century. The field is driven by, and often drives, the creation of new technology, including the development of high-precision and thermally-stable mechanical structures and the development of receiving elements approaching the quantum noise limit.

Millimetre-wave radio astronomy provides unique scientific contributions. Since the detection of interstellar formaldehyde $\left(\mathrm{H}_{2} \mathrm{CO}\right)$ in 1969 by Snyder, Buhl \& Zuckerman (1969), approximately 110 interstellar molecules have been identified by their line emissions in the millimetre and submillimetre wavebands (see Table 1), with more than 3,000 spectral lines observed, distributed more or less continuously in frequency except where oxygen absorption bands near 50 $\mathrm{GHz}, 118 \mathrm{GHz}$ and $183 \mathrm{GHz}$ prevent observation from the ground (Fig. 1).

\section{Telescope Time Usage}

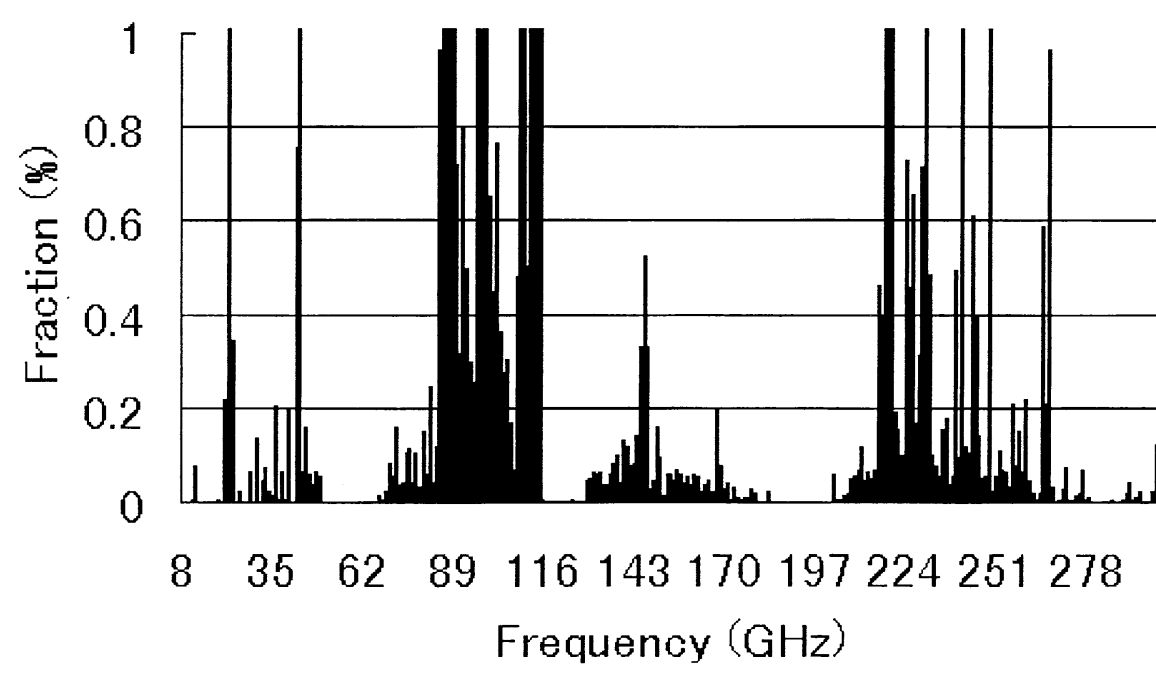

Figure 1. Statistics of the combined telescope time usage of the NRAO 12-m, Onsala 20-m, NRO 45-m, IRAM 30-m and SEST 15-m millimetre-wave radio telescopes. 
Table 1. Observed Interstellar Molecules as of July, 1999

Simple Hydrides, Oxides, Sulfides, Halogens and related molecules

$\begin{array}{lllll}\mathrm{H}_{2}(I R) & \mathrm{CO} & \mathrm{NH}_{3} & \mathrm{CS} & \mathrm{NaCl}^{*} \\ \mathrm{HCl} & \mathrm{SiO} & \mathrm{SiH}_{4}^{*}(I R) & \mathrm{SiS} & \mathrm{AlCl}^{*} \\ \mathrm{HF}(I R) & \mathrm{SO}_{2} & \mathrm{C}_{2}(I R) & \mathrm{H}_{2} \mathrm{~S} & \mathrm{KCl}^{*} \\ \mathrm{H}_{2} \mathrm{O} & \mathrm{OCS} & \mathrm{CH}_{4}^{*}(I R) & \mathrm{PN} & \mathrm{AlF}^{*}\end{array}$

$\mathrm{N}_{2} \mathrm{O}$

Nitriles and Acetylene derivatives

$\begin{array}{lllll}\mathrm{C}_{3}^{*}(I R) & \mathrm{HCN} & \mathrm{CH}_{3} \mathrm{CN} & \mathrm{HNC} & \mathrm{C}_{2} \mathrm{H}_{4}^{*}(I R) \\ \mathrm{C}_{5}^{*}(I R) & \mathrm{HC}_{3} \mathrm{~N} & \mathrm{CH}_{3} \mathrm{C}_{3} \mathrm{~N} & \mathrm{HNCO} & \mathrm{C}_{2} \mathrm{H}_{2}^{*}(I R) \\ \mathrm{C}_{3} \mathrm{O} & \mathrm{HC}_{5} \mathrm{~N} & \mathrm{CH}_{3} \mathrm{C}_{5} \mathrm{~N} & \mathrm{HNCS} & \\ \mathrm{C}_{3} \mathrm{~S} & \mathrm{HC}_{7} \mathrm{~N} & \mathrm{CH}_{3} \mathrm{C}_{2} \mathrm{H} & \mathrm{HNCCC} & \\ \mathrm{C}_{4} \mathrm{Si}^{*} & \mathrm{HC}_{9} \mathrm{~N} & \mathrm{CH}_{3} \mathrm{C}_{4} \mathrm{H} & \mathrm{CH}_{3} \mathrm{NC} & \\ & \mathrm{HC}_{11} \mathrm{~N} & \mathrm{CH}_{3} \mathrm{CH}_{2} \mathrm{CN} & \mathrm{HCCNC} & \\ & \mathrm{HC}_{2} \mathrm{CHO} & \mathrm{CH}_{2} \mathrm{CHCN}^{2} & & \end{array}$

Aldehydes, Alcohols, Ethers, Ketones, Amides and related molecules

$\begin{array}{lllll}\mathrm{H}_{2} \mathrm{CO} & \mathrm{CH}_{3} \mathrm{OH} & \mathrm{HCOOH} & \mathrm{CH}_{2} \mathrm{NH} & \mathrm{CH}_{2} \mathrm{CC} \\ \mathrm{H}_{2} \mathrm{CS} & \mathrm{CH}_{3} \mathrm{CH}_{2} \mathrm{OH} & \mathrm{HCOOCH}_{3} & \mathrm{CH}_{3} \mathrm{NH}_{2} & \mathrm{CH}_{2} \mathrm{CCC} \\ \mathrm{CH}_{3} \mathrm{CHO} & \mathrm{CH}_{3} \mathrm{SH} & \left(\mathrm{CH}_{3}\right)_{2} \mathrm{O} & \mathrm{NH}_{2} \mathrm{CN} & \\ \mathrm{NH}_{2} \mathrm{CHO} & \left(\mathrm{CH}_{3}\right)_{2} \mathrm{CO} & \mathrm{H}_{2} \mathrm{CCO} & & \\ & & \mathrm{CH}_{3} \mathrm{COOH} & & \end{array}$

Cyclic Molecules

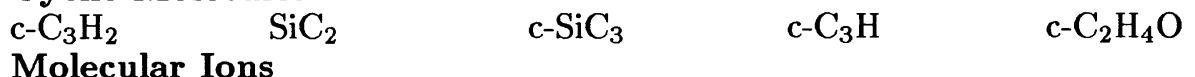

$\begin{array}{lllll}\mathrm{CH}^{+}(O P T) & \mathrm{HCO}^{+} & \mathrm{HCNH}^{+} & \mathrm{H}_{3} \mathrm{O}^{+} & \mathrm{HN}_{2}^{+} \\ \mathrm{HCS}^{+} & \mathrm{HOCO}^{+} & \mathrm{HC}_{3} \mathrm{NH}^{+} & \mathrm{HOC}^{+} & \mathrm{H}_{3}^{+} \\ \mathrm{CO}^{+} & \mathrm{H}_{2} \mathrm{COH}^{+} & \mathrm{SO}^{+} & & \\ \text {Radicals } & & & & \\ \mathrm{OH} & \mathrm{C}_{2} \mathrm{H} & \mathrm{CN} & \mathrm{C}_{2} \mathrm{O} & \mathrm{C}_{2} \mathrm{~S} \\ \mathrm{CH} & \mathrm{C}_{3} \mathrm{H} & \mathrm{C}_{3} \mathrm{~N} & \mathrm{NO} & \mathrm{NS} \\ \mathrm{CH}_{2} & \mathrm{C}_{4} \mathrm{H} & \mathrm{HCCN}^{*} & \mathrm{SO} & \mathrm{SiC}^{*} \\ \mathrm{NH}(U V) & \mathrm{C}_{5} \mathrm{H} & \mathrm{CH}_{2} \mathrm{CN} & \mathrm{HCO} & \mathrm{SiN}^{*} \\ \mathrm{NH}_{2} & \mathrm{C}_{6} \mathrm{H} & \mathrm{CH}_{2} \mathrm{~N} & \mathrm{MgNC} & \mathrm{CP}^{*} \\ \mathrm{HNO} & \mathrm{C}_{7} \mathrm{H} & \mathrm{NaCN} & \mathrm{MgCN} & \\ & \mathrm{C}_{8} \mathrm{H} & & & \end{array}$

For molecules observed in other frequency regions than radio, the wavebands are shown in parentheses : $I R$ - infrared, $O P T$ - optical and $U V$ - ultraviolet.

* - detected only in the envelope around the evolved star IRC+10216.

? - claimed but not yet confirmed. 
Such line emissions are used to study the star-formation process, the structure and formation of galaxies, and the process of star death. In general, this information is not available by any other means. In addition, broadband continuum emission arises in the millimetre wavebands from several physical mechanisms including free-free (bremsstrahlung) emission, synchrotron emission and the thermal emission from dust. The millimetre wavebands are critical for determining the spectral energy distribution of this emission, which in turn identifies the physical conditions of the emitting source.

Millimetre-wave radio astronomy places special requirements on the availability and usage of the frequency spectrum. These requirements fall in two broad categories: sensitivity and broad frequency bands. In general, radio astronomy at all frequencies requires extreme sensitivities, achieved by large, high-accuracy antennas, cryogenically-cooled electronics and long observations in which random noise is averaged to allow the detection of very weak cosmic signals. Millimetre-wave astronomy is certainly no exception to this requirement. Millimetre-wave astronomy has a nearly unique need for wide, interference-free bands. This requirement derives from four scientific and technical factors:

1. The ensemble of molecular rotational spectral line emission is distributed uniformly over the frequency spectrum. A few lines are observed more often than others, but many transitions of many species are required for the full and unique utilization of the science.

2. One of the most important applications of astronomical millimetre-wave spectroscopy is the observation of emission from gas in extremely distant, early-epoch galaxies. These studies are crucial to the understanding the evolution of galaxies and the early universe. Because of the expansion of the universe, the frequency at which this emission is received is reduced by factors ranging from a few percent (the local universe) to $80 \%$ (the currently-known earliest epochs of galaxy formation). Because of the continuous range of these redshifts, emission from important spectral lines can occur at any frequency in the millimetre wavebands.

3. Millimetre-wave continuum emission from galactic and extragalactic sources is typically exceedingly weak, but very important. Often, this emission can be detected only by using extremely wide instrumental bandwidths (up to $\sim 40 \mathrm{GHz}$ wide). In particular, incoherent bolometre detectors, currently the most sensitive detectors for $\mathrm{mm}$ and submm continuum work, require these very broad bandwidths to achieve their sensitivity.

4. The most sensitive instruments for $\mathrm{mm}$ and submm spectroscopy are SIS mixer receivers (Section 3.1), which respond to signals over bandwidths as large as $30 \%$ of their operating centre frequency. These exquisitely sensitive devices can be saturated or destroyed by a strong interfering signal anywhere in this bandwidth. Although it is possible to identify certain narrow frequency bands of very high importance to radio astronomy, the utilization of the full millimetre-wave spectrum is fundamental to the science. 


\section{Possible Radio Interference in the Near Future}

In the millimetre-wave regions several active services plan to utilize frequencies that may interfere with millimetre-wave observations. I show three examples below, but I note there are also other planned uses of the millimetre-wave bands by the military, etc.

\subsection{Collision Avoidance Radar at $76 \mathrm{GHz}$}

Vehicular radars are coming into operation in the band $76-81 \mathrm{GHz}$, which is allocated to the Radio Location Service on a primary basis. The third harmonic of the radar lies within the band $217-231 \mathrm{GHz}$ which is allocated to radio astronomy and other passive services on a primary basis. ITU-R Footnote S5.340 states that all emissions are prohibited within this band.

The proposed FCC standards on unwanted emissions above $200 \mathrm{GHz}$ are such that a coordination zone nearly $4 \mathrm{~km}$ in radius would be needed to avoid harmful interference to radio astronomy from just one single radar near an observatory. Most mm-wave observatories are not in a position to regulate vehicular traffic over such a large area. Indeed some are in areas of great interest to tourists and skiers. The radio astronomy community is concerned that the proliferation of these devices, coupled with possible degradation of their performance with age, will lead to an electromagnetic smog in the band $217-231 \mathrm{GHz}$ analogous to the light pollution which now compromises many once great optical observatories.

The radio astronomy community would like to propose that an on/off capability be incorporated into these devices so that, at the very least, $\mathrm{mm}$-wave observatories could place signs along nearby roads requesting that vehicular radar be turned off in the vicinity of the observatory. A better solution to the difficulty would be possible if industry could accept tighter specifications on unwanted emissions such that the coordination zone were less than $1 \mathrm{~km}$ in radius (within which most observatories have some control over traffic).

\subsection{HAPS near $31 \mathrm{GHz}$ and $47 \mathrm{GHz}$}

High Altitude Platform Systems (HAPS) have been proposed by the USA and Japan. The US system is proposed by a commercial company, Sky Station International (http://www.skystation.com/), and uses the band $47.2-47.5 \mathrm{GHz}$ for downlinks and the band 47.9-48.2 GHz for uplinks. Airships are to be located at an altitude of $\sim 21 \mathrm{~km}$ and more than 250 airships are planned, to cover every major city in the world. There are several important radio astronomy spectral lines near the $47-\mathrm{GHz}$ band : $\mathrm{CS}$ at $48.991 \mathrm{GHz}, \mathrm{CH}_{3} \mathrm{OH}$ at 48.372 and 48.377 $\mathrm{GHz}$, and $\mathrm{SiO}$ at $43.424,43.122 \mathrm{GHz}$ and so on, of which the $\mathrm{CS}$ and $\mathrm{SiO}$ bands are allocated to the radio astronomy service. Although ITU studies suggest that there will be no harmful interference from HAPS into the radio astronomy bands listed above, we need to be very careful to monitor developments.

Furthermore, Japan proposes to launch another HAPS system using bands at 19,29 and $31 \mathrm{GHz}$. Japan plans to launch more than 90 airships to cover almost all of Japan. Several airships will be visible from the Nobeyama Radio Observatory, where the $45-\mathrm{m}$ radio telescope and the Nobeyama Millimetre Array are located. The $31-\mathrm{GHz}$ HAPS band is $31.0-31.3 \mathrm{GHz}$, and the frequency 


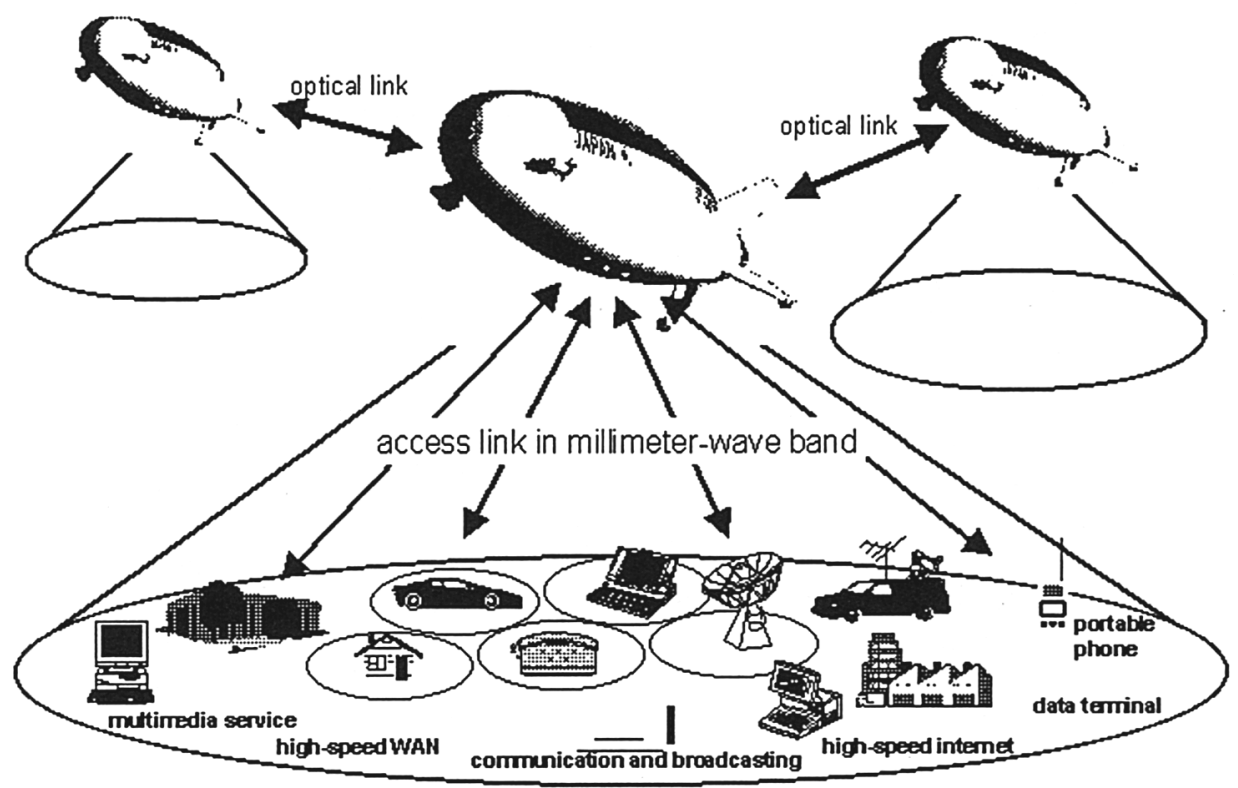

Figure 2. The HAPS system proposed by Japan

range is adjacent to an exclusive passive co-primary radio astronomy band, 31.3 $31.5 \mathrm{GHz}$, and a co-primary radio astronomy band, $31.5-31.8 \mathrm{GHz}$. These bands are used to measure cosmic microwave background radiation.

The Japanese system is more poisonous for us, because main beam - main beam couplings may occur between the radio telescope and the HAPS. In this case the received signal is estimated to be more than $150 \mathrm{~dB}$ above the harmful interference level when the airship is over the radio telescope! Even if the airship is over Tokyo the $45-\mathrm{m}$ telscope can detect the HAPS signal about $56 \mathrm{~dB}$ stronger than the harmful interference level. Therefore a guard band of $\sim 200 \mathrm{MHz}$ bandwidth is necessary in the HAPS band to protect millimetre-wave astronomy.

\subsection{Cloud Profiling Radar at $94 \mathbf{~ G H z}$}

WRC-97 allocated the band 94.0-94.1 GHz to the Earth Exploration Satellite (active) and Space Research (active) services and added Footnote S5.562: "The use of the band 94-94.1 GHz by the earth exploration-satellite (active) and space research (active) services is limited to space-borne cloud radars." The spaceborne cloud radars are downward-looking and will provide vertical sounding of cloud-layer structure down to the earth's surface.

The band $94.0-94.1 \mathrm{GHz}$ is close to several bands allocated to mm-wave radio astronomy:

$86-92 \mathrm{GHz}$ (Footnote $\mathrm{S} 5.340$, all emissions are prohibited in this band)

93.07 - 93.27 GHz (Footnote S5.149)

97.88 - $98.08 \mathrm{GHz}$ (Footnote S5.149)

Several potential interference mechanisms have been investigated. Unwanted emissions from the radar into the radio astronomy bands are not the only diffi- 
culty: indeed calculations suggest that with care the unwanted emissions can be kept below the radio astronomy interference thresholds. The main problem is how to protect the sensitive radio astronomy receiver from the powerful adjacent band radar signals. In practice the most common type of receiver used in mmwave observatories is the SIS mixer, which can saturate at very low power levels. Cloud radar transmissions could affect the operation of such receivers over their total frequency range, whenever the radio astronomy antenna is pointing within $14^{\circ}$ of the satellite, according to calculations based on preliminary antenna patterns. Furthermore, calculations suggest that an SIS mixer could actually be destroyed by a radar transmission directly into the main beam of the radio astronomy antenna.

Possible remedies to these problems have been investigated. It is technically very difficult to provide adequate filtering to radio astronomy receivers, to reject the cloud radar transmissions, while having very low loss so as not to compromise the receiver noise figure. The other main option is time sharing: either cover the radio telescope feed horn or switch off the radar transmissions whenever the satellite is above the observatory.

\section{Protection of Millimetre-Wave Astronomy}

\subsection{Technical Issues on Radio Astronomy Receivers}

1. Radio Astronomy Sensitivity Thresholds

General sensitivity thresholds for the Radio Astronomy Service are described in detail in ITU-R Recommendation RA. 769-1 and in the ITU-R Handbook on Radio Astronomy (Sections 4.3 - 4.6). The input power levels for detrimental interference are $\sim-185 \mathrm{dBW}$ for broadband continuum observations and $\sim-204 \mathrm{dBW}$ for narrow band spectral line observations.

\section{SIS Receiver Broadband Response}

Heterodyne mixer receivers utilizing SIS (Superconductor - Insulator - Superconductor) tunnel junctions are the devices of choice for modern mmand submm-wave spectroscopy. These devices are capable of noise performance approaching the quantum limit. The spectral bandwidth of such receivers can be up to a few $\mathrm{GHz}$ wide, but this width is usually limited by isolators and amplifiers following the mixer. A modern, tunerless SIS mixer can have efficient RF coupling over more than $30 \%$ of the band centre frequency as set by the mixer's local oscillator. A strong narrow-band system can overload an SIS mixer to the point of saturation or burnout (Pan et al., 1989).

\section{Continuum Bolometers}

Incoherent bolometers are the most sensitive detectors for broadband continuum emission in the millimetre and submillimetre bands. They are sensitive to photons with frequencies ranging from the infrared to the long millimetre wavelengths. To eliminate atmospheric noise and to define the wavelength band for detection, bolometers use bandpass filters. However, to maximize the bolometer sensitivity, the passbands typically cover the 
full width of an atmospheric window. Such wide frequency bandwidths can make bolometers vulnerable to RF interference. Weak, narrowband interference signals may be of little consequence because they will be diluted by the wide bandwidths of the bolometers. A strong CW signal may saturate a bolometer, however.

\section{Sidelobe Levels}

Interference in the main diffraction beam of an antenna sets the most stringent constraints on interference thresholds. Interference entering through the antenna sidelobes can also be a serious problem, as the sidelobes cover a much larger solid angle than the main lobe. Sidelobe response is also relevant to interference management schemes such as geographical sharing of the spectrum. A recommended sidelobe pattern of large parabolic antennas is given in Recommendation ITU-R SA. 509-1, which is applicable for frequencies between $2 \mathrm{GHz}$ and $30 \mathrm{GHz}$. The model for the envelope of the gain $(G)$ of the sidelobes is given by

$$
\begin{aligned}
& G=(32-25 \log \phi) \mathrm{dBi}, \quad 1^{\circ}<\phi<47.8^{\circ} ; \\
& G=-10 \mathrm{dBi}, \quad 47.8^{\circ}<\phi<180^{\circ} ;
\end{aligned}
$$

for angle $\phi$ measured from the axis of the main beam. It should be noted that the sidelobe pattern of the millimetre-wave antenna is similar to that of the centimetre-wave antenna.

\section{Atmospheric Propagation and Transparency}

Although molecular rotational emission is one of the great tools of millimetre-wave astronomy, some of the same kinds of transitions also cause absorption of cosmic signals as they traverse the earth's atmosphere. Rotational transitions of water vapour and spin-rotational transitions of molecular oxygen absorb signals to some extent at all millimetre-wave frequencies and completely stop the signal at certain frequencies. These absorption effects drive millimetre-wave observatories to high mountain-top locations where they are above as much of the atmospheric water vapour as possible. At some frequencies and in much of the submillimetre band, observatories must be located entirely above the earth's atmosphere in space-borne platforms. Indeed, some of the lines of $\mathrm{O}_{2}$ and $\mathrm{H}_{2} \mathrm{O}$ that form the strongest atmospheric absorption are also of great interest in cosmic sources. Although atmospheric absorption attenuates cosmic signals, it can also be used to advantage in shielding observatories from terrestrial interference. For the details of the atmospheric propagation, see, for example, Erickson \& Merino (1997).

\subsection{Possible Methods to Protect Millimetre-Wave Astronomy}

In millimetre and submillimetre-wave astronomy, a few frequency bands are of critical importance, but access to wide frequency bands is also fundamental to much of the science and technology. Frequency allocations above $30 \mathrm{GHz}$ must take this into account, or this field of astronomy will be seriously jeopardized. The radio astronomy community believes it possible to satisfy the needs of the radio astronomy service and the active services. The recommendations and strategies for future new allocations or reallocations are described below. 


\section{Reallocation of satellite down link frequencies}

Currently the frequency bands allocated to the radio astronomy service are sometimes surrounded by satellite downlink frequencies. This is a potential threat to the radio astronomy service owing to unintentional interference to sensitive wideband radio astronomy receivers. Reallocating satellite downlink bands to the edges of the atmospheric windows should be a compromise acceptable to the radio astronomy service: it would leave wide bands available for spectroscopy throughout the centre of the atmospheric windows, it would not affect the centre frequencies of the absorbing atmospheric lines, which may be of considerable importance to space-borne observatories and it may make it more feasible to construct high-pass or low-pass filters for receivers (as opposed to narrow notch filters). It should be noted that this arrangement will not come without cost to the radio astronomy service: some important spectral lines are near the edges of atmospheric windows and the construction of high-pass and low-pass filters may not be trivial.

\section{Co-Primary with other Passive Services}

Because all of the radio astronomy $\mathrm{mm}$-wave bands are passive, it is possible to share such bands with other passive services such as the Earth Exploration Satellite Services (passive). It is necessary to allocate these bands in the co-primary status to avoid potential interference from active services.

\section{Geographical and Time Sharing}

A further possibility for protection of the radio astronomy service is through use of geographical coordination zones and time-sharing schemes. There are relatively few millimetre and submillimetre observatories. Most are located at isolated, mountain-top sites. Consequently, geographical sharing of the spectrum is a possibility. In such a scheme, interfering ground-based transmissions would be prohibited in the vicinity of the observatory. Satellite downlinks could, in principle, be blanked as the transmission beam passed over the observatory. Ground-based transmissions in the vicinity of an observatory can be regulated by national administration, or possibly jointly with the administrations of the neighbouring countries, and do not necessarily require ITU agreements.

In time-sharing schemes, interfering transmitters would, by prior agreement, be turned off at certain times. This option is much less appealing than other allocation means because it restricts the time available for astronomy. Furthermore, it may be impractical for communication services to turn off their systems once the public becomes reliant on them. However, as a last resort, it could be used to protect $\mathrm{mm}$ and submm astronomy in certain geographical regions and/or for certain times of the day, or times of the year. 


\section{Conclusion}

The peaceful era for millimetre- and submillimetre-wave astronomy is now over. The radio astronomy service has been regarded as an eyesore for the active services, because radio astronomy needs very low interference threshold levels for its protection. How can we change this perception? I believe that we need to do more ourselves to advertise that radio astronomy is a very useful field of science that provides a lot of attractive new knowledge to human society. This is a very important activity that all radio astromoners can do, to help protect radio astronomy in the future.

Acknowledgments. I appreciate the work of all the members of the Millimetre-Wave Working Group (MMWG) of IUCAF, which was co-chaired by Phil Jewell of the Joint Astronomy Centre (JAC) and myself. This paper is based on the MMWG report presented at the ITU-R, adding several new topics.

\section{References}

Eriksson, J. E. P., Merino, F., 1997, "On Simulating Passive Observations of the Middle Atmosphere in the Range $1-1000 \mathrm{GHz}$ ", Research Report No. 179, Department of Radio and Space Science with Onsala Space Observatory, Chalmers University of Technology, Göteborg, Sweden

Pan, S.-K., Kerr, A. R., Feldman, M. J., Kleinsasser, A., Stasiak, J., Sandstrom, R. L., and Gallagher, W. J., 1989, IEEE Trans. Microwave Theory Tech., vol. MTT-37, no. 3,580

Snyder, L.E., Buhl, D., \& Zuckerman, B. 1969, Phys.Rev.Lett, 22, 679 